Mots. Les langages du politique

$94 \mid 2010$

Trente ans d'étude des langages du politique

(1980-2010)

\title{
Le corpus syndical. Une expérience au long cours
}

Sophie Béroud et Josette Lefèvre

\section{(2) OpenEdition}

Journals

Édition électronique

URL : https://journals.openedition.org/mots/19871

DOI : $10.4000 /$ mots. 19871

ISSN : 1960-6001

Éditeur

ENS Éditions

Édition imprimée

Date de publication : 30 novembre 2010

Pagination : 97-106

ISBN : 978-2-84788-235-3

ISSN : 0243-6450

Référence électronique

Sophie Béroud et Josette Lefèvre, "Le corpus syndical. Une expérience au long cours », Mots. Les langages du politique [En ligne], 94 | 2010, mis en ligne le 06 novembre 2012, consulté le 29 avril 2022 URL : http://journals.openedition.org/mots/19871 ; DOI : https://doi.org/10.4000/mots.19871 


\section{Mots \\ Les langages du politique}

№ 94 novembre 2010

\section{Trente ans d'étude des langages du politique (1980-2010)}

ouvrage coordonné par Paul BACOT, Marlène Coulomb-GulLY, Jean-Paul Honoré, Christian Le BART, Claire Oger, Christian Plantin

\section{SOMMAIRE}

Paul Bacot, Marlène Coulomb-Gully, Jean-Paul Honoré, Christian Le Bart, Claire Oger, Christian Plantin

Le discours politique n'est pas transparent. Permanence

et transformations d'un objet de recherche

OUTILS ET ENJEUX DU DISCOURS POLITIQUE

Ruth Amossy, Roselyne Koren

Argumentation et discours politique

Christian Plantin

Argumentation-rhétorique. Les eaux mêlées

Caroline Ollivier-Yaniv

Discours politiques, propagande, communication, manipulation 
Marc Bonhomme

La caricature politique

Paul Bacot

Développement et diversification d'une onomastique politique

Ruth Wodak

The Discursive Construction of History. Brief Considerations

Henri Boyer

Les politiques linguistiques

\section{LIEUX DE LA PRODUCTION DU DISCOURS POLITIQUE}

Christian Le Bart

Parler en politique

Dominique Maingueneau

Le discours politique et son «environnement»

Alice Krieg-Planque, Claire Oger

Discours institutionnels. Perspectives pour les sciences

de la communication

Sophie Béroud, Josette Lefèvre

Le corpus syndical. Une expérience au long cours

Corinne Gobin, Jean-Claude Deroubaix

L'analyse du discours des organisations internationales.

Un vaste champ encore peu exploré

François de la Bretèque

Le retour de la parole politique dans le cinéma français

Jean-Claude Soulages

Vie et mort du citoyen cathodique

Jacques Guilhaumou

Les discours de la Révolution française.

Aperçu d'ensemble d'un trajet de recherche (1980-2009) 


\section{DISCIPLINES ET CHAMPS DE RECHERCHE POUR L'ÉTUDE DES LANGAGES DU POLITIQUE}

Philippe Braud

L'apport de la science politique à l'étude des langages du politique

Claire Blandin

L'apport de l'histoire des médias à l'étude des langages du politique

Jean-François Tétu, Bernard Lamizet

Les SIC et les langages du politique

Sylvianne Rémi-Giraud

Sémantique lexicale et langages du politique.

Le paradoxe d'un mariage difficile?

Marlène Coulomb-Gully, Juliette Rennes

Genre, politique et analyse du discours.

Une tradition épistémologique française gender blind

Johannes Angermüller

Analyser le discours politique en Allemagne (1980-2010)

Érik Neveu

L'apport de Pierre Bourdieu à l'analyse du discours.

D'un cadre théorique à des recherches empiriques

Roselyne Ringoot

Questionner le discours avec Michel Foucault.

Actualisations théoriques et actualité éditoriale

\section{ENTRETIEN}

Maurice Tournier

Mots et politique, avant et autour de 1980

La revue Mots. Les langages du politique encourage l'usage des rectifications de l'orthographe proposées par le Conseil supérieur de la langue française et approuvées par l’Académie (Journal officiel, $n^{\circ}$ 100, 6 décembre 1990). 


\title{
Sophie Béroud \\ Josette Lefèvre
}

\section{Le corpus syndical. Une expérience au long cours}

\begin{abstract}
Double programme d'une sociohistoire des mots qui ne doit oublier ni le fait qu'ils sont serrés dans le microtissu d'actes énonciatifs ni le fait qu'ils font partie de la grande marche de l'histoire. Mariage difficile à harmoniser mais fructueux d'une lexicométrie du texte et d'une lexicologie de la société. Maurice Tournier (1989, p. 59).
\end{abstract}

La constitution d'un corpus syndical, rassemblant l'ensemble des résolutions votées lors des congrès de quatre confédérations interprofessionnelles (CFDT, CFTC, CGT, CGT-FO) depuis 1970, est étroitement liée à l'histoire du Centre de lexicologie de l'École normale supérieure de Saint-Cloud, plus connu sous le nom de laboratoire de «Lexicométrie et textes politiques ${ }^{1}$, même si la mise en forme et l'exploitation de celui-ci se sont prolongées au cours des années deux mille dans d'autres cadres institutionnels. Renvoyant à quatre décennies de travail collectif, ce corpus représente un outil unique et original qui permet de saisir de façon diachronique les inflexions du discours officiel des acteurs syndicaux, mais aussi sa porosité aux discours émis par d'autres locuteurs (gouvernement, patronat, institutions européennes et internationales...). Si les travaux menés sur la base de ce corpus ne résument pas à eux seuls l'état de la recherche sur le discours syndical, force est de constater que celle-ci demeure, par ailleurs, fragmentaire en France, ne donnant lieu qu'à des éclairages ponctuels. Le faible développement de ce sous-champ d'investigation rend, par contraste, d'autant plus importante l'élaboration dans la durée d'une base de données cohérente.

Université de Lyon, Université Lumière Lyon 2, Triangle

Sophie.Beroud@univ-lyon2.fr

Université Picardie Jules Verne, CNRS (CURAPP)

josette.lefevre@u-picardie.fr

1. Rapport d'activité 1977-1978 polycopié par l'ILF-Nancy pour le Comité de direction du 25 mai 1978. 


\section{La constitution du corpus. Enjeux méthodologiques et pratiques}

Sous la direction de Maurice Tournier, le laboratoire « Lexicométrie et textes politiques », placé à la jonction de la linguistique, de la statistique, de l'informatique et de la sociohistoire, se veut interdisciplinaire. Après un premier colloque international de lexicologie politique tenu en avril 1968, un «instrument lexicométrique » est mis au point; un ouvrage collectif, Des tracts en mai 1968. Mesures de vocabulaire et de contenu (Demonet et al., 1975) obtient une médaille de bronze du CNRS, ce qui vient conforter les options méthodologiques retenues. Érigée en dominante de recherche, la méthodologie lexicométrique va s'appliquer sur un corpus clos, élaboré à partir des hypothèses de recherche, y comptant toutes les formes graphiques comme des objets ordinaires, pour montrer que le dénombrable est significatif. Cette pratique lexicométrique va mobiliser l'ensemble des équipes constituées autour de corpus de recherche. Trois questions principales alimentent régulièrement les débats des séances du séminaire de recherche du laboratoire : Qu'est-ce que le politique? Qu'est-ce qu'un corpus? Qu'est-ce qu'un mot?

Les conditions matérielles contraignantes et rigides des années soixantedix laissent peu de place à l'expérimentation, ce qui a pu, parfois, influer sur la pertinence de certaines analyses. Les textes sont saisis, grâce à des crédits vacations alloués par un vote des chercheurs lors de l'assemblée générale annuelle, sur des perforatrices IBM 029 pour constituer les fichiers de données ou les fichiers programme (nécessité de refaire la carte en cas de faute de frappe). Les passages des programmes de calcul sur les données textuelles, pour obtenir les sorties-machines avec leurs listes de formes à dépouiller puis à analyser, dépendent d'informaticiens formés à l'utilisation du Centre de calcul. Entre le début d'une recherche et ses premiers résultats, plusieurs années souvent s'écoulent sans avoir la possibilité d'ajuster les choix de départ.

Lorsque, courant 1975, Maurice Tournier décide de former une équipe pour étudier le vocabulaire syndical, il réunit différents spécialistes du syndicalisme (politologues, historiens, linguistes). Certains, comme René Mouriaux, directeur de recherche à la Fondation nationale des sciences politiques, s'embarquent durablement dans l'aventure. Le choix de la réalisation d'un corpus clos constitué de toutes les résolutions votées en congrès confédéraux par les quatre organisations syndicales françaises ouvrières représentatives, interprofessionnelles et intercatégorielles que sont la CFDT, la CFTC, la CGT et Force Ouvrière entre 1971 et 1976, est pris après une étude argumentée. Ces résolutions discutées, voire amendées, avant d'être adoptées lors des assises confédérales font référence pour l'expression syndicale qui se diffuse ensuite dans 
l'organisation. Il s'agit du discours produit au «sommet» qui se présente de façon unifiée et comme un condensé des politiques syndicales s'imposant aux différentes structures de l'organisation. Outre son homogénéité discursive, cet aspect d'officialisation est majeur car il fonde le caractère représentatif de ce discours syndical. Ces résolutions apparaissent, en effet, comme dépositaires des usages lexicaux majoritaires qui se diffusent auprès des militants en responsabilité (responsables de fédérations, d'unions territoriales, de syndicats). Elles présentent une réelle homogénéité dans la mesure où elles constituent un matériau de nature comparable (des textes de résolutions pour l'activité à venir votées en congrès confédéral), s'adressant au même public (les délégués mandatés par leurs syndicats), dans des conditions d'énonciation similaire. Ces dimensions permettent d'asseoir une dimension comparative dans la durée, bien que le volume des textes votés par un congrès puisse varier d'une organisation à l'autre et dans le temps.

Le corpus représente dès 1976 un ensemble textuel de 146692 occurrences. Pour l'époque, c'est un «beau » et «gros» corpus. La publication de cette recherche pluridisciplinaire sous le titre La parole syndicale (Groupe de Saint-Cloud, 1982) fait date. Cependant, la note liminaire à cet ouvrage, visant à se prémunir des préjugés habituels à propos des analyses lexicométriques, fournit les explications suivantes:

Les mots et, au-delà les discours, sont dans l'histoire et ils sont des objets de l'analyse historique. En ce sens, la lexicométrie, comme les diverses formes d'analyse de discours ou de grammaire de texte, invite à rompre avec les descriptions qui considéraient mots et discours comme de pures transparences ou illustrations non problématiques de causes, thèses ou intérêts déclarés ou supposés. Et, en ce sens, elles remettent en question la problématique de la citation, toujours censée servir de preuve, sans que soit jamais interrogé ni même décrit l'univers linguistique complet où elle prend place. Nous voudrions donc porter un regard attentif sur les mots - tous les mots - du discours.

Toutefois, nous nous sommes refusés à considérer ces mots et liste ou suites de mots d'un point de vue strictement positiviste et à céder à la relative suspicion à l'égard de l'interprétation qu'on a pu reconnaître ici ou là dans certains travaux de lexicométrie, y compris ceux du laboratoire de Saint-Cloud. Nous avons, au contraire, proposé constamment des interprétations aux faits de fréquence, de spécificité, de cooccurrences que nous observions.

Mais loin de viser à restituer le savoir historique préalable, ces interprétations visent à fournir des explications aux faits de vocabulaire relevés.

C'est donc à l'histoire des mots - et des mots dans le discours - que nous entendons ici contribuer; car cette histoire est désormais partie intégrante, et nécessaire, de l'histoire tout court. (Groupe de Saint-Cloud, 1982, p.10-11) 


\section{Un corpus à l'épreuve du temps. Ce que montrent les variations d'emploi}

Au-delà de cette première publication collective, les outils informatiques s'étant simplifiés, un travail d'intégration régulier des résolutions votées en congrès confédéraux s'est poursuivi, permettant d'autres recherches originales, mais aussi facilitant diverses expérimentations méthodologiques. De fait, les choix des auteurs de La parole syndicale se trouvent aujourd'hui validés par le temps long de l'entreprise : approchant le million et demi d'occurrences, la base de données syndicales (1946-2010) fait référence pour tous ceux, et notamment les acteurs sociaux, qui s'intéressent au vocabulaire, à son histoire comme à son évolution.

L'exploitation d'un tel corpus permet plusieurs types d'approche, qu'il s'agisse de repérer les transformations des mouvements lexicaux d'ensemble au sein du vocabulaire syndical saisi dans sa globalité ou qu'il s'agisse de travailler à partir d'un découpage entre organisations, afin de saisir les mutations internes de chaque corpus confédéral et l'usage des termes qui font clivage entre les syndicats.

Ainsi, l'observation des variations d'emploi de quelques formes majeures du corpus syndical 1970-2010 partitionné en quatre décennies permet de prendre la mesure des évolutions lexicales qui se sont nécessairement opérées. Les dix premières formes lexicales de la décennie soixante-dix sont : travailleurs, travail, action, politique, congrès, conditions, développement, moyens, sociale et pays. Le remplacement de travailleurs par salariés au milieu des années quatrevingt entraîne la disparition de politique, congrès, conditions et moyens, formes auxquelles se substituent formation, entreprise et emploi. Dans le même mouvement, action rétrograde en huitième position et sociale passe de la neuvième à la sixième place. Les années quatre-vingt-dix entérinent la prépondérance de salariés, désormais au premier rang devant travail et sociale; la forme entreprises au pluriel apparaît en sixième position dans la liste. Des dix formes lexicales les plus fréquentes de la décennie soixante-dix, seules travail, congrès, sociale et développement sont encore présentes dans la dernière décennie².

Mais ces quatre formes ont changé de sens en même temps que d'environnement, comme l'ont montré d'autres études effectuées notamment sur le corpus CFDT, car «remplacer un terme par un autre, changer les systèmes d'alliances lexicales, c'est indissociablement s'opposer à ses discours

2. Les dix premières formes lexicales par partie et par ordre de fréquence sont les suivantes. Pour les années quatre-vingt : travail, salariés, travailleurs, développement, formation, sociale, entreprise, action, pays, emploi. Années quatre-vingt-dix : salariés, travail, sociale, emploi, développement, congrès, formation, entreprises, action, entreprise. Années deux mille : salariés, travail, congrès, sociale, emploi, entreprises, entreprise, droits, développement, organisation. 
antérieurs [...]» (Groupe de Saint-Cloud, 1982, p. 233; Lafon, Lefèvre, 1997). Le recours aux concordances s'avère indispensable et précieux, comme le montrent quelques exemples. Ainsi, en première période, l'adjectif sociale se trouve accolé à un ensemble de substantifs porteurs d'une analyse politique de la société : appropriation sociale des moyens de production, conscience sociale et politique des travailleurs, division sociale du travail. Mais dans la dernière décennie, c'est par d'autres lexies qu'il s'insère dans un paysage différent : protection sociale, responsabilité sociale, insécurité sociale, sécurité sociale professionnelle. Le substantif développement connaît le même dépaysement : pays en développement, développement des luttes, nouveau type de développement, mode de développement capitaliste, inégalités de développement, développement économique marquent les années soixante-dix, quand d'autres lexies ou syntagmes sans recoupement possible sont emblématiques des décennies ultérieures : développement de l'emploi, développement de politiques actives, développement des opportunités de temps choisi, développement de la négociation interprofessionnelle locale, secteurs en développement, développement de la syndicalisation, développement durable.

L'analyse lexicométrique du corpus TSYND-1971-1976 avait conduit les auteurs de La parole syndicale à cette conclusion :

Dans le syndicalisme français, ce sont donc les divisions idéologiques qui paraissent l'emporter. En tout cas, l'éclatement des deux traditions, laïque et chrétienne, qui l'ont constitué à ses origines est manifeste et leur redistribution s'opère schématiquement selon une opposition entre un "syndicalisme de négociation» et un "syndicalisme de contestation»; mais ces deux nouveaux pôles n'ont pas engendré d'unité idéologique et ne déterminent stratégiquement que des convergences incomplètes.

Quelques années plus tard, dans sa préface au Dictionnaire des fréquences établi sur le corpus TSYND-1971-1990, Antoine Prost, sous le titre «Syndicalisme de classe ou gestion d'intérêts sociaux», prévenait : "Évitons cependant de durcir l'opposition des deux groupes de syndicats : la situation est plus fluide. Le vocabulaire de classe et de combat n'est suremployé à la CFDT et à la CGT que durant les années soixante-dix. Des évolutions se sont produites, qui demandent examen. » (Hetzel et al., 1998, p. 9) Examinons à la lumière de ces questionnements les spécificités les plus fortes de chacune des quatre décennies qui composent le corpus TSYND-1970-2010.

Les formes qui caractérisent le plus les années soixante-dix, en opposition radicale avec les trois autres décennies donc, sont les termes représentatifs du lexique du syndicalisme de lutte de classe et de contestation de la société développé par la CFDT et la CGT : travailleurs, classe, capitaliste, socialiste, socialisme, monopoles, action, politique, capitalisme, exploitation, pouvoir, masse, lutte, conditions, démocratique, sociétés multinationales, forces, forces populaires, système capitaliste, société socialiste, société capitaliste, 
programme commun, démocratie économique (et politique), ensemble des travailleurs, libertés (syndicales), luttes, masses, unité d'action, anticapitaliste, équipements collectifs, immigrés, lutte de classe, économique et politique, autogestionnaire, capitalistes, Tiers-Monde, Viêt-Nam, gauche. Toutes ces formes sont spécifiées négativement dans la dernière décennie.

Le poids lexical de la CGT pèse sur les années quatre-vingt. Avec trois congrès volubiles et redondants tenus dans la décennie, le sous-corpus CGT compte, en effet, deux fois plus d'occurrences que les textes réunis des trois autres confédérations; en conséquence, les formes les plus spécifiques de cette partie proviennent de la panoplie lexicale qu'elle déploie, isolément, sur le front de la déstabilisation de l'économie française menacée selon elle par la «mondialisation des entreprises», les délocalisations industrielles et l'intégration européenne: pays, crise, transnationales, casse, gâchis, coopérations, reconquête du marché intérieur, production(s), intervention des travailleurs/ salariés, capital, besoins, secteur public, modernisation, nouvelle croissance, technologies nouvelles, changement.

Face au doute existentiel qui assaille la CGT et à la tourmente interne qui déstabilise la CFDT au cours des années quatre-vingt-dix, la CFTC se lance à la reconquête d'adhérents sigle en tête : CFTC, communication, personnes, valeurs, vivre, familiale occupent le haut de la liste. Le sigle CGT-FO s'affiche aussi alors que le pronom nous est mobilisé par la CFDT comme par la CGT. Plus ou moins communs aux quatre centrales, d'autres termes portent les inquiétudes syndicales dans un monde où les inégalités sociales progressent et se cristallisent: syndicalisme, salariés, exclusion, citoyenneté, emploi, précarité, défi, monde du travail. Dans un tel contexte d'impuissance, la dimension européenne se présente comme une opportunité à saisir : Europe, européenne, européen (Lefèvre, 2006) participent de l'européanisation du discours confédéral avec l'importation de termes et de syntagmes nominaux en usage à la Confédération européenne des syndicats (CES).

Enfin, la liste des formes ou lexies spécifiques des années 2000-2010 donne à voir l'intégration d'un vocabulaire nouveau, structurant, révélateur d'un renforcement de l'institutionnalisation du syndicalisme avec une implication de plus en plus grande de l'acteur syndical dans la construction et la gestion de la démocratie sociale : congrès, dialogue social, CFDT, salariés, statut du travailleur, territoires, négociation, syndiqués, acteurs, adhérents, syndicalisation, statut du travail salarié, responsabilité sociale, syndicat(s), accompagnement, gouvernance, fonctions publiques, attentes, épargne salariale, cotisations, parcours professionnel(s), MEDEF, compétences, sociale, démocratie sociale, cohésion sociale, retraite, demandeurs d'emploi, marché du travail, salariat, représentativité, partenaires sociaux, mondialisation, services publics, développement durable... Un vocabulaire de négociation sociale qui n'est pas plus propre à l'univers syndical que ne l'était celui de lutte de classe. 


\section{Rendre compte de l'univers syndical au travers de différents genres de discours}

L'étude des contextes, que rend également possible le corpus TSYND-1970-2010, passe par la recontextualisation des unités étudiées, des cooccurrences. Le choix d'une entrée thématique, par un mot, contribue à voir comment une lexie peut recouvrir des sens différents dans le temps et à restituer une part des stratégies discursives des acteurs. La démarche sert dès lors, pour le lexicologue mais aussi pour le politiste, à entrer dans l'univers conceptuel des syndicats français (D’Almeida, Mouriaux, 1993), dans leurs représentations du monde social et de la place qu'ils y occupent. Les pratiques discursives, telles qu'elles sont consolidées dans les congrès, contribuent à orienter les pratiques militantes vers ce qu'il semble possible de faire et de revendiquer, donnant du sens à l'action (Gagnon, 1991). Des entrées thématiques ont ainsi été privilégiées, centrées sur la façon de dire le groupe qu'il s'agit de représenter (Lefèvre, 2003) ou, autre exemple, sur les façons de concevoir la démocratie dans le monde du travail et dans l'entreprise (Béroud, Lefèvre, 2007). À chaque fois, il s'agit de restituer la spécificité du vocabulaire syndical, mais aussi de comprendre les emprunts et la circulation d'un vocabulaire émis par d'autres locuteurs. La constitution en cours d'un corpus du vocabulaire patronal3 viendra de ce point de vue combler un manque et enrichir les connaissances dans ce domaine.

Ces perspectives de recherche, qui s'efforcent de restituer la façon dont la parole syndicale se construit en interaction avec d'autres visions du monde et s'inscrit dans de multiples controverses (à la fois internes au champ syndical et liées à l'actualité politique et sociale), sont privilégiées dans des travaux qui s'intéressent à des genres de discours distincts de la résolution de congrès. Du côté des spécialistes du syndicalisme, l'étude de la presse des différentes organisations peut ainsi servir de point d'appui pour comprendre la construction des argumentaires face aux réformes gouvernementales (Erbes-Seguin, 1973; Verdier, 1981; Siwek-Pouydesseau, 2010). Le genre du discours étudié ainsi que le support retenu délimitent de façon forte la portée de l'analyse et ce qu'il est possible d'interpréter. Il est vrai que les argumentaires présents dans la presse syndicale «ne sont pas toujours lus par les militants, et encore moins par les adhérents. Cependant, leur répétition finit par imprégner la culture syndicale et sert à définir les identités des différentes organisations [...] 》 (SiwekPouydesseau, 2010, p. 746). Mathieu Brugidou a proposé une approche théorique plus systématisée du genre de discours qu'est la revendication syndicale (Brugidou, 2000). Reprenant la définition de la revendication comme « un droit

3. Projet de recherche de la DARES du ministère du Travail sur les « Organisations d'employeurs en France» (2009-2011). 
non encore reconnu ou obtenu », mais aussi «l'action de réclamer une chose» (Bernard-Steindecker, 1993), il insiste sur la dimension performative de celleci. Une des propriétés particulières du discours syndical provient dès lors du «travail rhétorique » qui consiste à « effacer la distance entre la représentation et l'action » (Brugidou, 2000, p. 968) tout en produisant un cadre cognitif susceptible de rassembler les salariés par-delà la diversité de leurs situations et de leurs intérêts. À partir de cette problématique, Mathieu Brudigou étudie les éditoriaux de la presse syndicale entre 1996 et 1998 (optant pour les quatre confédérations initialement retenues par l'équipe de Saint-Cloud) et recourt à une méthode d'analyse textuelle statistique avec le logiciel Alceste.

Le fait de se porter vers d'autres genres de discours - le tract (Burtin, 1977; Diani, Bagnara, 1984), les comptes rendus de réunion (Gardin, 1984), l'éditorial de presse, l'allocution, etc. - permet de saisir dans sa complexité une parole syndicale qui n'est pas dissociable du contexte dans lequel elle est produite, de la finalité qui lui est conférée et de l'espace dialogique dans lequel elle évolue. Mais il est à noter, d'une part, que cette diversité dans les supports retenus et dans les options méthodologiques, présente dans les années soixantedix et quatre-vingt (Drigeard, Mouriaux, 1987), s'est réduite dans les décennies suivantes, faute d'une production scientifique suffisante. Les supports écrits, d'autre part, ne résument pas à eux seuls ce qui constitue la parole syndicale, la façon dont elle s’incarne dans des activités sociales. Bernard Gardin et Josiane Boutet se sont ainsi intéressés à une activité de langage plus routinière, saisie dans des interactions ordinaires liées au quotidien du travail (Boutet, Gardin, Lacoste, 1995). Loin d'être formalisées d'une certaine manière par l'institution syndicale, les données sont ici largement construites, dans une démarche proche de l'ethnométhodologie, via le dispositif d'observation mis en place par le chercheur. D'une façon différente, puisque s'appuyant sur un corpus clos constitué d'une trentaine d'entretiens recueillis auprès de militants syndicaux investis au niveau européen, Anne-Catherine Wagner a analysé la très forte dépendance de la parole syndicale par rapport au vocabulaire technique présent dans les différentes productions de la Commission européenne (Wagner, 2008). Mais il s'agit là encore d'un déplacement intéressant puisque c'est le récit de vie, marqué par l'oralité et l'interaction avec le chercheur, qui sert de matériau empirique.

Outre l'expérience au long cours du corpus syndical, qui constitue un outil original pour rendre compte, sur plus de quatre décennies, de la structuration interne du discours syndical, le champ d'étude que constitue le discours syndical se révèle aujourd'hui faiblement investi. Plusieurs connexions seraient pourtant intéressantes à établir pour enrichir celui-ci, qu'il s'agisse d'étudier le vocabulaire dans la sphère du travail, l'emprise du champ lexical du management (Borzeix, Fraenkel, 2001) ou de continuer à diversifier les approches méthodologiques pour rendre compte de l'inscription de la parole syndicale dans des pratiques sociales différenciées, plus ou moins institutionnalisées. 


\section{Références}

BernaRd-SteindeCKer C., 1993, "Vingt ans de revendication», Mots. Les langages du politique, $\mathrm{n}^{\circ} 36$, septembre, p. 48-60.

Béroud S., Lefèvre J. éd., 2003, Mots. Les langages du politique, nº71, mars, Mondialisation(s).

BÉRoud S., LEFÈVRE J., 2007, «Vers une démocratie économique et sociale? Banalisation et redéploiement du vocabulaire syndical», Mots. Les langages du politique, no 83 , mars, p. 37-52.

Borzeix A., Fraenkel B. éd., 2001, Langage et travail. Communication, cognition, action, Paris, CNRS.

Boutet J., GARdin B., LACOSte M., 1995, «Discours en situation de travail», Langages, vol.XXIX, n' ${ }^{0} 117$, p.12-31.

BRUGIDOU M., 2000, "Les discours de la revendication et de l'action dans les éditoriaux de la presse syndicale (1996-1998)», Revue française de science politique, vol. L, n०6, p. 967-992.

BURTIN P., 1977, «Attitudes et idéologies syndicales. Une analyse de tracts distribués à Renault Billancourt», Revue française de science politique, vol.XXVII, nº 6, p. 915-931.

D’Almeida F., Mouriaux R., 1993, "Syndicats entre État et entreprises», Mots. Les langages du politique, $\mathrm{n}^{\circ} 36$, septembre, p. 6-17.

DEMONET M. et al. éd., 1975, Des tracts en mai 1968, Paris, FNSP/Armand Colin.

DIANI M., BAGNARA S., 1984, "Les tracts comme indicateurs de tensions conflictuelles », Revue française de sociologie, vol.XXV, n³ 3, p. 376-395.

DRIGEARD G., MouriauX R., 1987, «Bibliographie sur le vocabulaire et le discours syndical français contemporain », Mots. Les langages du politique, n014, mars, p. 199-202.

ERBÈs-SEgUIN S., 1973, «La presse syndicale en 1971. Thèmes professionnels et économiques », Sociologie du travail, n ${ }^{4}$, p. 376-395.

GAGNON M.-J., 1991, «Le syndicalisme. Du mode d'appréhension à l'objet sociologique », Sociologies et sociétés, vol.XXIII, n² 2, p.79-95.

GARDIN B., 1984, "Un récit d'interaction. Les comptes rendus de délégation syndicale », Langages, vol.XIX, nº74, p. 93-122.

Groupe de SAINT-CLOUd, 1982, La parole syndicale. Étude du vocabulaire confédéral des centrales ouvrières françaises, 1971-1976, Paris, PUF.

Hetzel A.-M., Lefèvre J., Mouriaux R., Tournier M., 1998, Le syndicalisme à mots découverts. Dictionnaire des fréquences (1971-1990), Paris, Syllepse.

Hetzel A.-M., Mouriaux R., Tournier M. éd., 1993, Mots. Les langages du politique, $n^{0} 36$, septembre, Un demi-siècle de vocabulaire syndical.

LAFON P., LEFÈVRE J., 1997, «Le figement. Prise en compte discursive, incidences sur les statistiques textuelles et sur l'interprétation », La locution. Entre lexique, syntaxe et pragmatique, P. Fiala et al. éd., Paris, Klincksieck, p. 295-306.

LEFÈVRE J., 2003, «De l'univers lexical de travailleurs à celui de salariés. La CFDT de 1970 à 1998 : évolution d'un parcours identitaire», Constructions identitaires et dynamiques politiques, L. Baugnet éd., Bruxelles, Peter Lang, p. 193-211. 
- 2006, "Le vocabulaire international de la CGT (1972-2003)», La place et les actions de la CGT dans le monde (1945-2000), E. Bressol éd., Montreuil, Institut CGT d’histoire sociale, p.71-86.

SiWek-Pouydesseau J., 2010, "Les syndicats de la fonction publique et les réformes managériales depuis 2002 ", Revue française d'administration publique, no 132 , p. $745-756$.

Tournier M., 1989, "Pour une sociohistoire des mots-conflits», Courants sociolinguistiques. Séminaire de lexicologie politique de l'Université de Paris 3 (1986-1987), P. Fiala et al. éd., Paris, Klincksieck, p. 53-62.

Tournier M., LefèVre J. éd., 1987, Mots. Les langages du politique, no ${ }^{14}$, mars, Discours syndical ouvrier en France.

VERDIER E. et al., 1981, La presse syndicale ouvrière. Analyse statistique de contenu, Sceaux, CRESST.

WAGNER A.-C., 2008, «L'expertise syndicale européenne. L'imbrication des logiques de l'expertise et du discours militant chez les syndicalistes européens », Mots. Les langages du politique, $\mathrm{n}^{\circ} 88$, novembre, p. 53-65. 